\title{
Changes in choroidal thickness in advanced diabetic retinopathy treated with pan- retinal photocoagulation using a pattern scanning laser versus a conventional laser
}

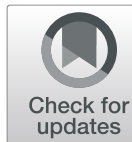

Nari Park', In Gul Lee ${ }^{2}$ and Jee Taek Kim ${ }^{1 *}$

\begin{abstract}
Background: To compare the effect of pan-retinal photocoagulation (PRP) using pattern scanning or conventional laser on subfoveal choroidal thickness (SFChT).

Methods: Thirty-eight patients (64 eyes) with advanced diabetic retinopathy (DR) who underwent PRP using pattern scanning or conventional laser were included. Changes in SFChT were compared with baseline values at 1 , 3,6 , and 12 months after PRP using swept-source optical coherence tomography.

Results: The conventional laser group showed a statistically significant decrease in SFChT at 1, 3, 6, and 12 months after PRP $(P<0.001)$. SFChT was significantly decreased at $3(P=0.025), 6(P=0.004)$, and $12(P<0.001)$ months after treatment in the pattern laser group.

Conclusion: Eyes with advanced DR showed a significant reduction in SFChT over 12 months regardless of the type of laser used; however, the reduction was sooner after conventional laser than after pattern laser.

Keywords: Choroidal thickness, Conventional laser, Diabetic retinopathy, Optical coherence tomography, Pan-retinal photocoagulation, Pattern scanning laser
\end{abstract}

\section{Background}

Diabetic retinopathy (DR) is a common complication of diabetes and a leading cause of vision loss in workingaged populations in both advanced and developing countries [1]. Pan-retinal photocoagulation (PRP) is considered the gold standard treatment for severe nonproliferative diabetic retinopathy (NPDR) and proliferative retinopathy (PDR) according to the Early Treatment Diabetic Retinopathy Study (ETDRS) [2]. Traditionally, PRP has been performed using various wavelengths, including argon green $(514 \mathrm{~nm})$ or diode $(810 \mathrm{~nm})$ lasers.

\footnotetext{
* Correspondence: jeetaek-kim@hanmail.net

'Department of Ophthalmology, College of Medicine, Chung-Ang University Hospital, 102 Heukseok-ro, Dongjak-gu, Seoul 06974, South Korea

Full list of author information is available at the end of the article
}

However, these lasers have recently been replaced by a pattern scanning laser system that uses a frequencydoubled $532 \mathrm{~nm}$ wavelength Nd:YAG laser to avoid patient discomfort and fatigue. Furthermore, the reduced pulse duration required when using the pattern scanning laser may be less painful because of decreased thermal diffusion into the choroid [3].

Several studies have shown that SFChT is affected by treatment, including PRP and intravitreal injections [4-9]. However, there have been no studies comparing the effect of conventional laser and pattern scanning laser systems on choroidal thickness. It has been reported that PRP performed with the pattern scanning laser is less effective than that performed with a traditional laser for regression of retinal

(C) The Author(s). 2020 Open Access This article is licensed under a Creative Commons Attribution 4.0 International License, which permits use, sharing, adaptation, distribution and reproduction in any medium or format, as long as you give appropriate credit to the original author(s) and the source, provide a link to the Creative Commons licence, and indicate if changes were made. The images or other third party material in this article are included in the article's Creative Commons licence, unless indicated otherwise in a credit line to the material. If material is not included in the article's Creative Commons licence and your intended use is not permitted by statutory regulation or exceeds the permitted use, you will need to obtain permission directly from the copyright holder. To view a copy of this licence, visit http://creativecommons.org/licenses/by/4.0/. The Creative Commons Public Domain Dedication waiver (http://creativecommons.org/publicdomain/zero/1.0/) applies to the data made available in this article, unless otherwise stated in a credit line to the data. 
neovascularization in eyes with high-risk PDR [10]. The purpose of this study was to compare the effect of PRP on choroidal thickness using the conventional and pattern scanning lasers.

\section{Methods}

\section{Subjects}

This retrospective interventional comparative study was approved by the institutional review board committee of Chung-Ang University Hospital, Seoul, South Korea, and adhered to the tenets of the Declaration of Helsinki. The medical records of consecutive patients who underwent PRP for PDR or severe NPDR at the Chung-Ang University Hospital from September 1, 2015 to July 31, 2017 and who were followed up for at least 12 months were retrospectively reviewed. A signed informed consent was obtained from all patients before each PRP session. The exclusion criteria included systemic disease other than diabetes, prior retinal surgery or photocoagulation, any history of eye disease, retinal disease or choroidal disease, a history of ocular trauma, and refractive error $\geq \pm 3.0$ diopters.

Furthermore, patients who received anti-vascular endothelial growth factor (VEGF) injection before the baseline date and during the follow-up period were excluded, as were patients who underwent cataract surgery within 6 months before the baseline date and during follow-up.

Eyes with low-quality OCT images (an image quality index $<90$ ) because of media opacities such as vitreous hemorrhage or cataract were additionally excluded. However, eyes with good-quality OCT images and localized vitreous hemorrhage or peripheral cortical lens opacity were not excluded.

All patients underwent a comprehensive ophthalmic examination, an fluorescein angiography, and sweptsource OCT (DRI Triton OCT, Topcon, Tokyo, Japan). The severity of DR, central retinal thickness (CRT), and SFChT were determined by previously described methods [11].

\section{Pan-retinal photocoagulation}

PRP was done by a single retinal specialist (JTK) with a $532 \mathrm{~nm}$ frequency-doubled neodymium-doped yttrium aluminum garnet (Nd-YAG) solid-state pattern scan laser (Valon pattern laser, Dual Laser Ltd. Oy, Finland) or a $532 \mathrm{~nm}$ solid-state green diode laser (OcuLight GLx laser, Iridex Corp. Mountain View, CA, USA). The patients were classified into a pattern laser group or a conventional laser group as appropriate. Patients underwent PRP with a conventional diode laser before January 2017 and PRP with a pattern laser from January 2017 onwards after acquisition of the new laser. PRP was placed from the superior and inferior vascular arcades to the peripheral retina and performed in all eyes in both groups in two sessions one week apart.

For pattern laser, a $5 \times 5$ multispot array with a $200 \mu \mathrm{m}$ spot size, a $20 \mathrm{~ms}$ pulse duration, and a 1.5 width spot spacing was used. The laser power was adjusted from $200 \mathrm{~mW}$ until a gray-white opacity was achieved. For conventional laser, the laser was performed by previously described [11].

\section{Statistical analysis}

The data are expressed as the mean \pm standard deviation. The CRT, SFChT, reduction in SFChT $(\triangle \mathrm{SFChT})$, and rate of reduction in SFChT $(\% \triangle \mathrm{SFChT})$ were analyzed during follow-up. Statistical analyses were done using SPSS version 23.0 software (IBM Corp., Armonk, NY, USA) with the independent $t$-test, paired $t$-test, and chisquare test as appropriate. A $P$-value $<0.05$ was considered statistically significant.

\section{Results}

\section{Baseline characteristics}

Sixty-four eyes (38 patients) with severe NPDR $(n=27)$ or PDR $(n=37)$ were included in this study. There were 37 eyes in the conventional laser group and 27 eyes in the pattern laser group. The mean patient age was $55.2 \pm 11.7$ (range 34-71) years, and the mean $\mathrm{HbA}_{1 \mathrm{c}}$ value was $8.0 \% \pm 1.8 \%$, the mean duration of diabetes was $13.2 \pm 5.8$ (range 7-22) years. The mean BCVA was $0.24 \pm 0.25 \log M A R$, the mean spherical equivalent was $-0.73 \pm 1.8$, and the mean intraocular pressure was $15.2 \pm 3.3 \mathrm{mmHg}$. There was no significant difference in age, sex, refractive error, $\mathrm{HbA}_{1 \mathrm{c}}$, BCVA, or mean duration of diabetes between the two study groups (Table 1).

PRP was performed using a mean power of $318.5 \pm 285.7$ (range 210-500) $\mathrm{mW}$ in the conventional laser group and a mean power of $355.9 \pm$ 111.5 (range 240-540) $\mathrm{mW}$ in the pattern laser group $(P=0.061)$. The total number of photocoagulation burns was $1512.8 \pm 246.8$ in the conventional laser group and $3216.8 \pm 287.3$ in the pattern laser group $(P<0.001)$.

There was no correlation between the mean baseline SFChT and age $(P=0.138), \mathrm{HbA}_{1 \mathrm{c}}(P=0.237)$, BCVA $(P=0.747)$, or spherical equivalent $(P=0.795)$. The inter-observer reproducibility of the SFChT measurement ranged from 0.986 to 0.990 . The baseline CRT was similar in both groups and there was no statistically significant difference in the changes in CRT following PRP between the study groups. 
Table 1 Demographic and clinical characteristics of patients included in the study

\begin{tabular}{lllll}
\hline & Total (64 eyes) & Conventional laser group (37 eyes) & Pattern laser group (27 eyes) & $P$-value \\
\hline Age (years) & $55.2 \pm 11.7$ & $55.3 \pm 11.3$ & $55.1 \pm 12.7$ & $0.936^{\mathrm{a}}$ \\
Sex M/F (no.) & $37 / 27$ & $22 / 15$ & $15 / 12$ & $0.755^{\mathrm{b}}$ \\
$\mathrm{HbA}_{1 c}$ (\%) & $8.0 \pm 1.8$ & $8.3 \pm 2.2$ & $7.8 \pm 1.5$ & $0.33^{\mathrm{a}}$ \\
Duration of DM (years) & $13.2 \pm 5.8$ & $13.1 \pm 4.8$ & $13.3 \pm 6.7$ & $0.56^{\mathrm{a}}$ \\
BCVA (logMAR) & $0.24 \pm 0.25$ & $0.27 \pm 0.29$ & $0.21 \pm 0.2$ & $0.486^{\mathrm{a}}$ \\
IOP (mmHg) & $15.2 \pm 3.3$ & $14.2 \pm 3.6$ & $16.2 \pm 2.6$ & $0.068^{\mathrm{a}}$ \\
SE (diopter) & $-0.73 \pm 1.8$ & $-0.63 \pm 1.8$ & $15 / 12$ & $0.944^{\mathrm{a}}$ \\
PDR/Severe NPDR (no.) & $37 / 27$ & $22 / 15$ & $250.3 \pm 67.4$ & $0.477^{\mathrm{b}}$ \\
Baseline CRT ( $\mu \mathrm{m})$ & $266.2 \pm 87.4$ & $284.1 \pm 104.7$ & $0.166^{\mathrm{a}}$ \\
\hline
\end{tabular}

$B C V A$ best-corrected visual acuity, $C R T$ central retinal thickness, $D M$ diabetes mellitus, $H b A_{1 c}$ glycated hemoglobin, IOP intraocular pressure, $N P D R$ non-proliferative retinopathy, PDR proliferative retinopathy, SE spherical equivalent

andependent $\mathrm{t}$-test; ${ }^{\mathrm{b}}$ chi-square test

\section{Mean subfoveal choroidal thickness in the conventional and pattern laser groups}

The mean SFChT in the conventional laser group was $318.4 \pm 58.3 \mu \mathrm{m}$ at baseline and decreased significantly $(P<0.001$ vs baseline $)$ at $1,3,6$ and 12 months, respectively (Fig. 1a).

The mean SFChT in the pattern laser group was $313.3 \pm 91.9 \mu \mathrm{m}$ at baseline. There were no significant changes in the mean SFChT between baseline and 1 month $(309.5 \pm 93.4 \mu \mathrm{m}, P=0.404$ vs baseline) after PRP. The mean SFChT were significantly decreased to $309.5 \pm 93.4 \mu \mathrm{m}$ at 3 months ( $P=0.404$ vs baseline), $301.2 \pm 89.6 \mu \mathrm{m}$ at 6 months $(P=0.004$ vs baseline $)$, and $285.1 \pm 87.6 \mu \mathrm{m}$ at 12 months $(P<0.000$ vs baseline; Fig. 1a).

$\triangle$ SFChT was significantly greater in the conventional laser group than in the pattern laser group at 1 month $(14.7 \pm 17.3 \mu \mathrm{m}$ vs $3.8 \pm 21.9 \mu \mathrm{m}, P=0.03)$, 3 months $(20.8 \pm 18.6 \mu \mathrm{m}$ vs $9.4 \pm 19.1 \mu \mathrm{m}, P=0.027)$, and 6 months $(25.3 \pm 21.2 \mu \mathrm{m}$ vs $12.1 \pm 18.5 \mu \mathrm{m}, P=$ $0.018)$ after PRP. There was no statistically significant difference at 12 months $(38.3 \pm 19.2 \mu \mathrm{m} \quad$ vs $28.1 \pm 22.8 \mu \mathrm{m}, \quad P=0.075$ ) after PRP (Fig. 1b, Table 2).

The $\% \triangle \mathrm{SFChT}$ was also greater in the conventional laser group than in the pattern laser group at 1 month $(4.5 \% \pm 5.1 \%$ vs $1.1 \% \pm 7.2 \%, P=0.52), 3$ months $(6.3 \% \pm$ $5.5 \%$ vs $2.7 \% \pm 6.4 \%, P=0.03), 6$ months $(7.8 \% \pm 6.7 \%$ vs $3.7 \% \pm 6.4 \%, P=0.02)$, and 12 months $(11.9 \% \pm 5.9 \%$ vs $8.9 \% \pm 7.2 \%, P=0.087$; Table 2).

\section{Changes in subfoveal choroidal thickness between the severe NPDR and PDR groups}

Thirty-seven of the 64 eyes (38 patients) had PDR and 27 had severe NPDR. The mean SFChT in the PDR group was $319.4 \pm 73.5 \mu \mathrm{m}$ at baseline and decreased significantly through the follow-up period after PRP. The mean SFChT in the severe NPDR group was $309.1 \pm 74.3 \mu \mathrm{m}$ at baseline and also decreased significantly at every time point after PRP (Fig. 2a).

The $\triangle$ SFChT values in the PDR and severe NPDR groups were $9.9 \pm 22.3 \mu \mathrm{m}$ and $11.7 \pm 15.5 \mu \mathrm{m}$, respectively, at 1 month, $16.7 \pm 20.9 \mu \mathrm{m}$ and $13.7 \pm 16.8$ at 3 months, and $24.6 \pm 22.5$ and $20.9 \pm 16.2$ at 6 months. There was no statistically significant difference in the amount of reduction in $\triangle \mathrm{SFChT}$ between the two groups. However, the $\triangle \mathrm{SFChT}$ was significantly greater in the PDR group than in the severe NPDR group at 12 months after PRP $(38.6 \pm 22.9 \mu \mathrm{m}$ vs $24.8 \pm 13.7, P=$ 0.006; Fig. 2b; Table 2).

The $\% \triangle \mathrm{SFChT}$ values in the PDR and severe NPDR groups were $2.7 \% \pm 6.9$ and $3.6 \% \pm 4.8 \%$, respectively, at 1 month, $4.9 \% \pm 6.5$ and $4.4 \% \pm 5.2 \%$ at 3 months, and $7.6 \% \pm 7.3$ and $7.3 \% \pm 5.6 \%$ at 6 months. There was no statistically significant difference in the amount of change in $\% \triangle S F C h T$ between the two groups. However, the $\% \triangle \mathrm{SFChT}$ was significantly greater in the PDR group than in the severe NPDR group at 12 months after PRP $(12.1 \% \pm 7.3 \%$ vs $8.1 \% \pm 4.2 \%, P=0.01$; Table 2$)$.

\section{Discussion}

In this study, we compared the changes in SFChT in eyes with advanced DR after PRP between a conventional laser group and a pattern laser group. There was a significant decrease in SFChT after PRP compared with the baseline in both groups of eyes during the follow-up period. The reduction in SFChT was more prominent in the conventional laser group than in the pattern laser group at an early stage following PRP.

Blumenkranz et al. have developed a pattern scanning laser with a shorter pulse duration of $10-20 \mathrm{~ms}$ that uses a Nd:YAG laser with a $532 \mathrm{~nm}$ wavelength [3]. They reduced the pulse duration of the laser to decrease patient discomfort and to reduce the amount of time taken to 


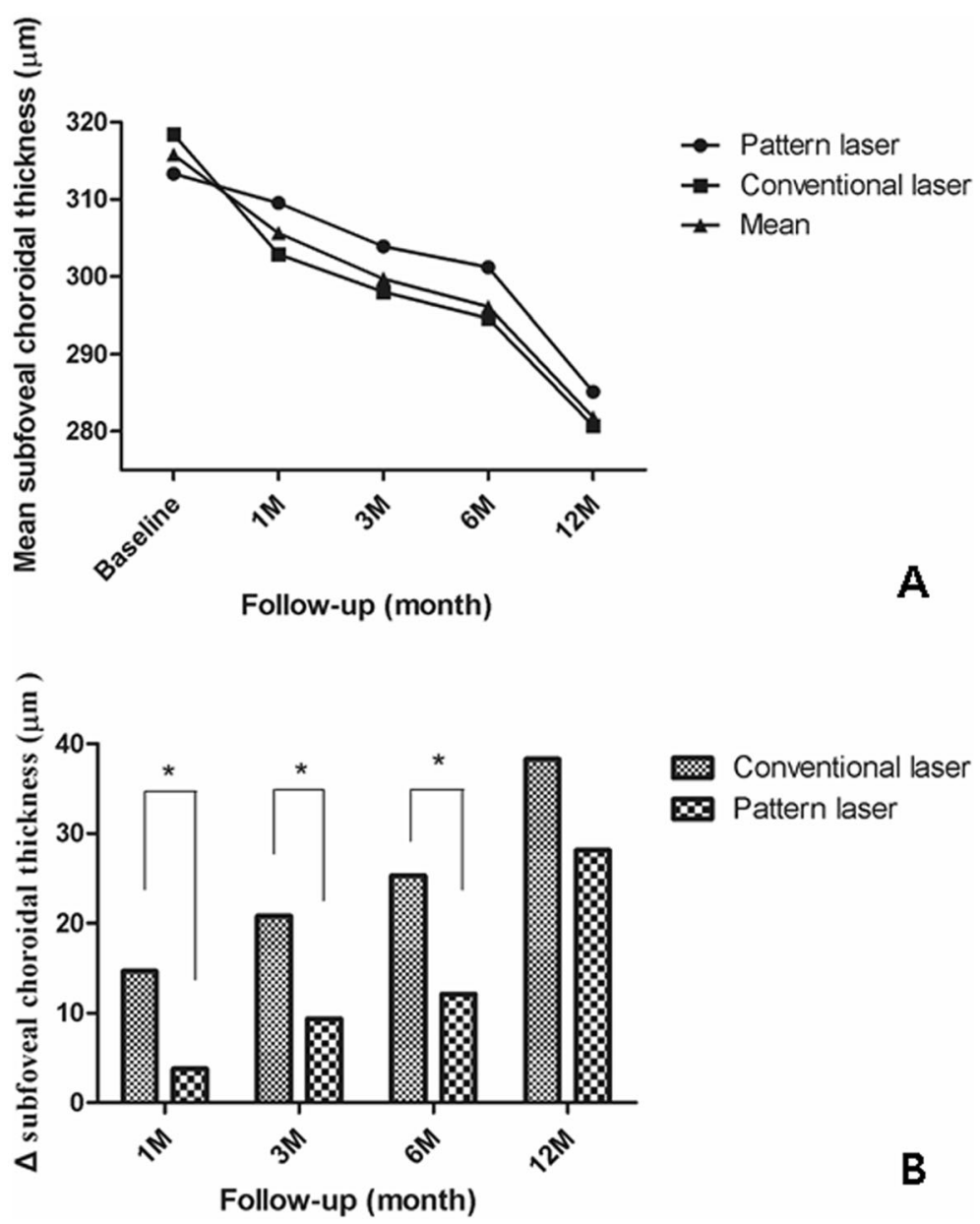

Fig. 1 Comparison of the changes and reduction in SFChT after pan-retinal photocoagulation between the conventional laser and pattern laser groups. a Comparison of changes in mean SFChT between the two groups. The mean SFChT was significantly decreased during follow-up. $\mathbf{b}$ Comparisons of the reduction in SFChT between the two groups. The reduction was more prominent in the conventional laser group than in the pattern group, especially in the early period after pan-retinal photocoagulation. SFChT, mean subfoveal choroidal thickness; $\Delta$ SFChT, reduction of SFChT $=$ SFChT at baseline - SFChT at time point. ${ }^{*} P<0.05$. SFChT, subfoveal choroidal thickness

perform PRP. The shorter pulse duration requires less pulse energy. The laser scars produced by the shorter pulses are smaller than those produced by longer pulses [12]. Nagpal et al. reported that the average scar size created by the conventional laser was significantly greater than that created by the pattern scanning laser $(430 \mu \mathrm{m}$ vs $310 \mu \mathrm{m})$ at 3 months after PRP with the same $200 \mu \mathrm{m}$ spot size [13]. Photocoagulation scars created using a conventional laser with a pulse duration of $100-200 \mathrm{~ms}$ tend to enlarge progressively because of heat diffusion [12]. Therefore, it seems that the difference in the changes in SFChT between the two groups after PRP in this study was related to differences in the expansion of the laser burn scar. Moreover, the laser fluence created by the pattern scanning laser was significantly less than that created by the conventional laser $\left(40.33 \mathrm{~J} / \mathrm{cm}^{2}\right.$ vs $191 \mathrm{~J} / \mathrm{cm}^{2}$ ) [13].
Several studies have shown that the SFChT decreases after PRP and that PRP-treated eyes have a smaller SFChT than naïve eyes $[4,8,9,14-16]$. Zhang et al. found that the SFChT decreased significantly 3 months after PRP [8], as did Okamoto et al. [9], while Ohara et al. found that SFChT decreased significantly 6 months after PRP [16] and Kang et al. reported a significant reduction in the SFChT 12 months after PRP [14]. The present study also showed that the SFChT was significantly reduced over 12 months after PRP. This finding is consistent with the earlier research with regards to a long-lasting PRP effect [14]. Previously, Maeshima et al. described progressive enlargement of the laser scar over several years [17]. Moreover, they reported that the mean annual laser scar expansion rates were $7-12.7 \%$. We suggest that the long-lasting effect on SFChT over 12 
Table 2 Comparison of mean subfoveal choroidal thickness between the two study groups during follow-up after pan-retinal photocoagulation

\begin{tabular}{|c|c|c|c|c|c|c|c|}
\hline & \multirow[b]{2}{*}{$\begin{array}{l}\text { Total }(n= \\
64)\end{array}$} & \multicolumn{3}{|c|}{ Changes in mean SFChT according to laser used } & \multicolumn{3}{|c|}{ Changes in mean SFChT according to severity of DF } \\
\hline & & $\begin{array}{l}\text { Pattern laser group ( } n= \\
\text { 27) }\end{array}$ & $\begin{array}{l}\text { Conventional laser group } \\
(n=37)\end{array}$ & $\begin{array}{l}{ }^{\mathrm{a}} P_{-} \\
\text {value }\end{array}$ & $\begin{array}{l}\text { Severe NPDR group ( } n= \\
\text { 27) }\end{array}$ & $\begin{array}{l}\text { PDR group }(n= \\
37)\end{array}$ & $\begin{array}{l}{ }^{a} P_{-} \\
\text {value }\end{array}$ \\
\hline $\begin{array}{l}\text { SFChT } \\
\text { Baseline }\end{array}$ & $315.8 \pm 73.3$ & $313.3 \pm 91.9$ & $318.4 \pm 58.3$ & 0.798 & $309.1 \pm 74.3$ & $319.4 \pm 73.5$ & 0.617 \\
\hline SFChT $1 \mathrm{M}$ & $305.6 \pm 71.6$ & $309.5 \pm 93.4$ & $302.9 \pm 52.7$ & 0.742 & $297.4 \pm 72.5$ & $310.1 \pm 71.8$ & 0.527 \\
\hline SFChT $3 \mathrm{M}$ & $299.7 \pm 69.6$ & $303.9 \pm 89.6$ & $298.0 \pm 52.3$ & 0.758 & $295.4 \pm 72.7$ & $302.1 \pm 68.8$ & 0.73 \\
\hline SFChT $6 \mathrm{M}$ & $296.1 \pm 72.6$ & $301.2 \pm 89.6$ & $294.6 \pm 57.9$ & 0.74 & $290.2 \pm 74.4$ & $296.0 \pm 72.6$ & 0.993 \\
\hline SFChT $12 \mathrm{M}$ & $281.8 \pm 69.4$ & $285.1 \pm 87.6$ & $280.7 \pm 53.9$ & 0.819 & $284.2 \pm 71.6$ & $280.4 \pm 69.1$ & 0.847 \\
\hline$\Delta$ SFChT $1 \mathrm{M}$ & $10.1 \pm 20.1$ & $3.8 \pm 21.9$ & $14.7 \pm 17.3$ & 0.03 & $11.7 \pm 15.5$ & $9.3 \pm 22.3$ & 0.666 \\
\hline$\Delta$ SFChT $3 \mathrm{M}$ & $16.1 \pm 19.5$ & $9.4 \pm 19.1$ & $20.8 \pm 18.6$ & 0.027 & $13.7 \pm 16.9$ & $17.3 \pm 20.9$ & 0.519 \\
\hline$\Delta$ SFChT $6 \mathrm{M}$ & $19.7 \pm 21.0$ & $12.1 \pm 18.5$ & $25.3 \pm 21.2$ & 0.018 & $12.9 \pm 16.2$ & $23.4 \pm 22.5$ & 0.072 \\
\hline$\Delta \mathrm{SFChT} 12 \mathrm{M}$ & $34.0 \pm 21.2$ & $28.1 \pm 22.8$ & $38.3 \pm 19.2$ & 0.075 & $24.8 \pm 13.7$ & $38.9 \pm 22.9$ & 0.006 \\
\hline $\begin{array}{l}\% \Delta \text { SFChT } 1 \\
M\end{array}$ & $2.9 \pm 6.2$ & $1.1 \pm 7.2$ & $4.5 \pm 5.1$ & 0.52 & $3.7 \pm 4.8$ & $2.6 \pm 6.9$ & 0.525 \\
\hline $\begin{array}{l}\% \Delta \text { SFChT } 3 \\
M\end{array}$ & $4.8 \pm 6.1$ & $2.7 \pm 6.4$ & $6.3 \pm 5.5$ & 0.03 & $4.4 \pm 5.3$ & $5.0 \pm 6.6$ & 0.701 \\
\hline $\begin{array}{l}\% \Delta \text { SFChT } 6 \\
M\end{array}$ & $6.2 \pm 6.8$ & $3.7 \pm 6.4$ & $7.8 \pm 6.7$ & 0.02 & $4.2 \pm 5.6$ & $7.2 \pm 7.3$ & 0.121 \\
\hline $\begin{array}{l}\% \Delta \text { SFChT } 12 \\
M\end{array}$ & $10.7 \pm 6.6$ & $8.9 \pm 7.2$ & $11.9 \pm 5.9$ & 0.087 & $8.1 \pm 4.2$ & $12.2 \pm 7.3$ & 0.01 \\
\hline
\end{tabular}

NPDR non-proliferative retinopathy, PDR proliferative retinopathy, SFChT subfoveal choroidal thickness, $\triangle S F C h T$ reduction of SFChT SFChT at baseline - SFChT at time point, $\% \triangle S F C h T$ percentage of reduction in SFChT $=100 \times($ SFChT at baseline - SFChT at time point)/SFChT at baseline

andependent $t$-test

months is associated with expansion of the laser burn scar.

Despite the consistent finding of a decrease in the SFChT during long-term observation, reports on the more short-term studies have been conflicting but suggest a significant increase in SFChT at 1-12 weeks after PRP [18-20]. It is thought that the inconsistent early changes in SFChT after PRP might be caused by release of inflammatory cytokines in response to laser photocoagulation. Four eyes in our pattern laser group and two eyes in our conventional laser group showed thickening of the SFChT by more than $5 \%$ $\triangle \mathrm{SFChT}$. It seems that eyes that received higher laser energies are more likely to have thickened SFChT in the early phase after PRP than eyes that received lower laser energies. Thus, we think that a subgroup analysis according to different laser energies or glycemic control status would be interesting. However, the number of eyes was too small to obtain statistically significant results.

We also compared the changes in SFChT after PRP between the PDR and severe NPDR groups. The two groups showed a similar tendency for a decrease in SFChT during the study period. However, the reduction in SFChT was more prominent in the PDR group than in the severe NPDR group at 12 months following PRP. The reduction in SFChT after PRP may be caused by a decrease in the level of vascular endothelial growth factor (VEGF) secreted from the non-perfusion area in response to ablation of an ischemic retina and choriocapillaris. There have been reports of a significant reduction in SFChT in eyes treated with intravitreal injections of anti-VEGF or triamcinolone acetonide as well as PRP [21-23]. These findings also suggest that the choroid tissue is sensitive to the amount of VEGF or cytokines released from the retinal tissue.

Essentially, we have attempted to analyze the correlation between the change in DR stage and SFChT after PRP. Before PRP, the DR stage was usually evaluated by FAG. However, FAG is not regularly performed to analyze the regression of new vessels in eyes with proliferative DR and rarely performed in eyes with severe NPDR after PRP. Therefore, a prospective study will be needed to analyze the correlation between the regression of DR stage and the changes in SFChT after PRP.

This study has several limitations. First, is its small sample size. The second is its retrospective rather than controlled prospective design. Third, recurrence or persistence of new vessels in eyes with PDR after PRP was not considered. Fourth, eyes with severe or high-risk PDR were not included in this study, because most received additional PRP or vitrectomy due 

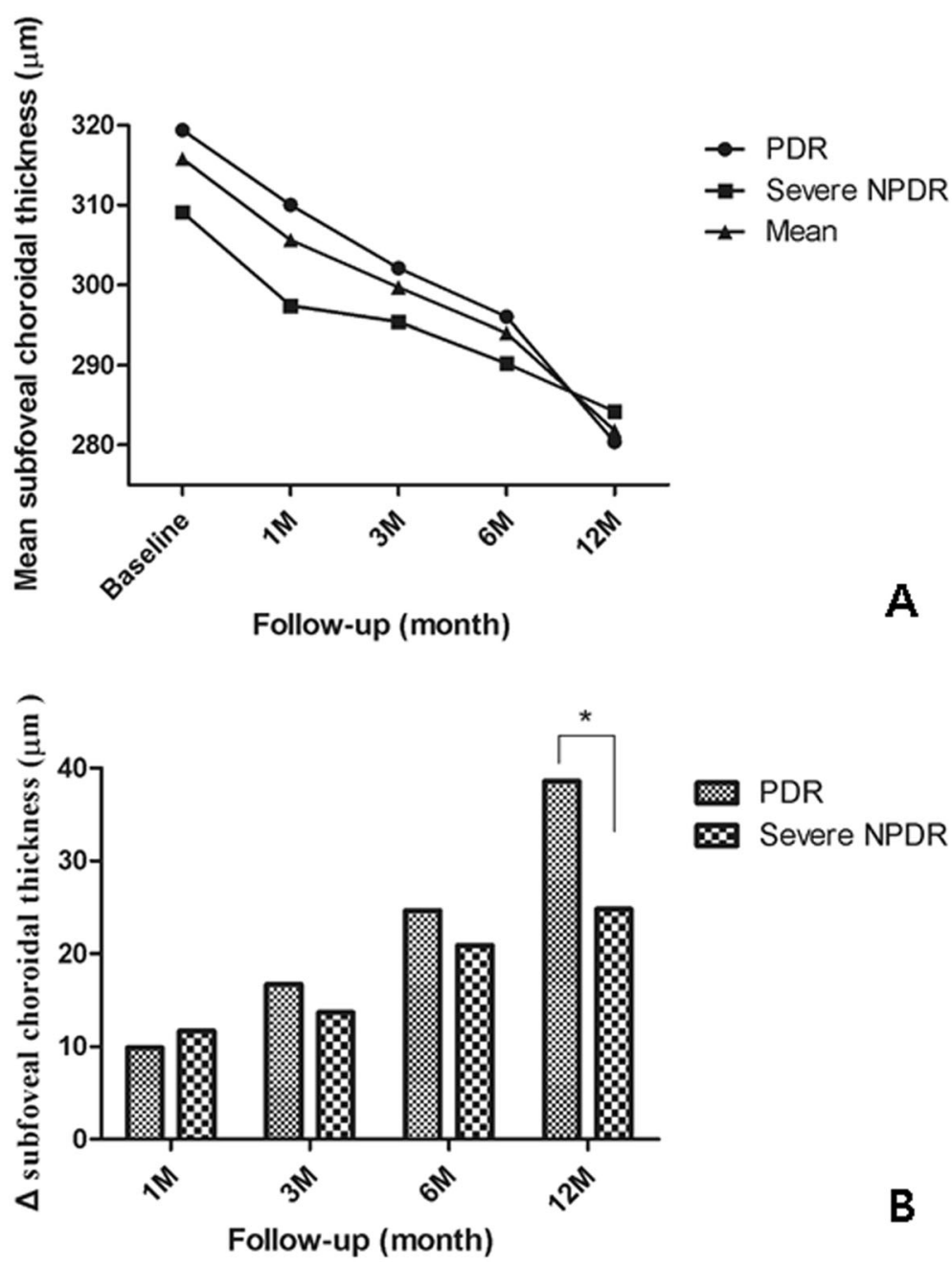

Fig. 2 Comparisons of the changes and reduction in mean subfoveal choroidal thickness (SFChT) after pan-retinal photocoagulation between the PDR and severe NPDR groups. a Comparison of changes in mean SFChT between the two groups. $\mathbf{b}$ Comparison of the reduction in SFChT between the two groups. The PDR group showed a more prominent reduction than the severe NPDR group at 12 months after pan-retinal photocoagulation. $\triangle \mathrm{SFChT}$, reduction of SFChT $=$ SFChT at baseline - SFChT at time point. PDR, proliferative retinopathy; NPDR, non-proliferative retinopathy $* P=0.006$

to persistent new vessels or vitreous hemorrhage. Fifth, diurnal variation was not considered. Sixth, the effect of intravitreal anti-VEGF injection was not considered in eyes with DME. However, despite these limitations, this is the first study to compare the effect of pattern scanning laser with that of conventional laser on SFChT after PRP. Further investigations with a prospective design are needed to confirm the findings of the present study.

\section{Conclusion}

SFChT is reduced significantly after PRP. However, the effect of conventional laser on the SFChT is greater than that of pattern scanning laser, especially in the early stages after PRP.

\section{Abbreviations}

BCVA: best-corrected visual acuity; CRT: central retinal thickness; DR: diabetic retinopathy; ETDRS: Early Treatment Diabetic Retinopathy Study; NdYAG: neodymium-doped yttrium aluminum garnet; NPDR: non-proliferative diabetic retinopathy; PDR: proliferative retinopathy; PRP: pan-retinal photocoagulation; SFChT: subfoveal choroidal thickness; $\triangle$ SFChT: reduction in SFChT; \% $\triangle \mathrm{SFChT}$ : rate of reduction in SFChT

\section{Acknowledgments}

Not applicable.

Authors' contributions

Involved in the design of the study (JTK); conduct of the study (JTK); collection, management, analysis of the data (NP, JTK); preparation of the manuscript (NP, JTK, IGL); and critical revision of the manuscript (JTK, IGL). All authors read and approved the final manuscript.

\section{Funding}

Following are results of a study on the "Leaders in INdustry-university Cooperation +" Project, supported by the Ministry of Education and National Research Foundation of Korea. This work was supported by the National 
Research Foundation of Korea (NRF) grant funded by the Korea government (Ministry of Science and ICT) (No. 2020R1F1A1072226). These funding sources had no role in the study design; collection, analysis, or interpretation of the data or in the writing of the report.

\section{Availability of data and materials}

The datasets used and/or analyzed in this study are available from the corresponding author on reasonable request.

\section{Ethics approval and consent to participate}

The study protocol was approved by the IRB committee of Chung-Ang University Hospital, Seoul, South Korea (IRB no. 1701-003-16029) and was conducted in accordance with the tenets of the Declaration of Helsinki. Signed informed consent was obtained from all patients before each PRP treatment session.

\section{Consent for publication}

Not applicable.

\section{Competing interests}

The authors declare that they have no competing interests.

\section{Author details}

'Department of Ophthalmology, College of Medicine, Chung-Ang University Hospital, 102 Heukseok-ro, Dongjak-gu, Seoul 06974, South Korea. ${ }^{2}$ Dangjin bright eye center, Dangjin, South Korea.

\section{Received: 18 April 2019 Accepted: 2 June 2020}

Published online: 12 June 2020

\section{References}

1. Zheng Y, He M, Congdon N. The worldwide epidemic of diabetic retinopathy. Indian J Ophthalmol. 2012;60:428-31.

2. Early Treatment Diabetic Retinopathy Study Research Group. Early photocoagulation for diabetic retinopathy. ETDRS report number 9 . Ophthalmology. 1991:98:766-85.

3. Blumenkranz MS, Yellachich D, Andersen DE, Wiltberger MW, Mordaunt D, Marcellino GR, Palanker D. Semiautomated patterned scanning laser for retinal photocoagulation. Retina (Philadelphia, Pa). 2006;26:370-6.

4. Kim JT, Lee DH, Joe SG, Kim JG, Yoon YH. Changes in choroidal thickness in relation to the severity of retinopathy and macular edema in type 2 diabetic patients. Invest Ophthalmol Vis Sci. 2013;54:3378-84.

5. Regatieri CV, Branchini L, Carmody J, Fujimoto JG, Duker JS. Choroidal thickness in patients with diabetic retinopathy analyzed by spectral-domain optical coherence tomography. Retina (Philadelphia, Pa). 2012;32:563-8.

6. Gerendas BS, Waldstein SM, Simader C, Deak G, Hajnajeeb B, Zhang L, Bogunovic H, Abramoff MD, Kundi M, Sonka M, Schmidt-Erfurth U. Threedimensional automated choroidal volume assessment on standard spectraldomain optical coherence tomography and correlation with the level of diabetic macular edema. Am J Ophthalmol. 2014;158:1039-48.

7. Vujosevic S, Torresin T, Berton M, Bini S, Convento E, Midena E. Diabetic macular edema with and without Subfoveal Neuroretinal detachment: two different morphologic and functional entities. Am J Ophthalmol. 2017:181:149-55.

8. Zhang Z, Meng X, Wu Z, Zou W, Zhang J, Zhu D, Chen T, Zhang Q. Changes in Choroidal thickness after Panretinal photocoagulation for diabetic retinopathy: a 12-week longitudinal study. Invest Ophthalmol Vis Sci. 2015;56:2631-8.

9. Okamoto M, Matsuura T, Ogata N. Effects of panretinal photocoagulation on choroidal thickness and choroidal blood flow in patients with severe nonproliferative diabetic retinopathy. Retina (Philadelphia, Pa). 2016;36:805-11.

10. Chappelow AV, Tan K, Waheed NK, Kaiser PK. Panretinal photocoagulation for proliferative diabetic retinopathy: pattern scan laser versus argon laser. Am J Ophthalmol. 2012:153:137-42 e132.

11. Kim JT, Park N. Changes in choroidal vascular parameters following panretinal photocoagulation using swept-source optical coherence tomography. Graefes Arch Clin Exp Ophthalmol. 2020;258:39-47.

12. Jain A, Blumenkranz MS, Paulus Y, Wiltberger MW, Andersen DE, Huie $P$, Palanker D. Effect of pulse duration on size and character of the lesion in retinal photocoagulation. Archives Ophthalmol (Chicago, III : 1960). 2008; 126:78-85.

13. Nagpal M, Marlecha S, Nagpal K. Comparison of laser photocoagulation for diabetic retinopathy using 532-nm standard laser versus multispot pattern scan laser. Retina (Philadelphia, Pa). 2010;30:452-8.

14. Kang HM, Lee NE, Choi JH, Koh HJ, Lee SC. Significant reduction of both peripapillary and subfoveal choroidal thickness after panretinal photocoagulation in patients with type 2 diabetes. Retina (Philadelphia, Pa). 2018;38:1905-12.

15. Lee SH, Kim J, Chung H, Kim HC. Changes of choroidal thickness after treatment for diabetic retinopathy. Curr Eye Res. 2014;39:736-44.

16. Ohara Z, Tabuchi H, Nakakura S, Yoshizumi Y, Sumino H, Maeda Y, Kiuchi Y. Changes in choroidal thickness in patients with diabetic retinopathy. Int Ophthalmol. 2018;38:279-86.

17. Maeshima K, Utsugi-Sutoh N, Otani T, Kishi S. Progressive enlargement of scattered photocoagulation scars in diabetic retinopathy. Retina (Philadelphia, Pa). 2004;24:507-11.

18. Cho GE, Cho HY, Kim YT. Change in subfoveal choroidal thickness after argon laser panretinal photocoagulation. Int J Ophthalmol. 2013;6:505-9.

19. Roohipoor R, Dantism S, Ahmadraji A, Karkhaneh R, Zarei M, Ghasemi F. Subfoveal Choroidal thickness after Panretinal photocoagulation with red and green laser in bilateral proliferative diabetic retinopathy patients: short term results. J Ophthalmol. 2016;2016:9364861.

20. Zhu Y, Zhang T, Wang K, Xu G, Huang X. Changes in choroidal thickness after panretinal photocoagulation in patients with type 2 diabetes. Retina (Philadelphia, Pa). 2015;35:695-703.

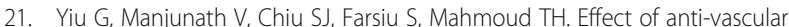
endothelial growth factor therapy on choroidal thickness in diabetic macular edema. Am J Ophthalmol. 2014;158:745-51 e742.

22. Lains I, Figueira J, Santos AR, Baltar A, Costa M, Nunes S, Farinha C, Pinto R, Henriques J, Silva R. Choroidal thickness in diabetic retinopathy: the influence of antiangiogenic therapy. Retina (Philadelphia, Pa). 2014;34:1199-207.

23. Sonoda S, Sakamoto T, Yamashita T, Otsuka H, Shirasawa M, Kakiuchi N, Uchino $\mathrm{E}$, Terasaki $\mathrm{H}$, Kawano $\mathrm{H}$. Effect of intravitreal triamcinolone acetonide or bevacizumab on choroidal thickness in eyes with diabetic macular edema. Invest Ophthalmol Vis Sci. 2014;55:3979-85.

\section{Publisher's Note}

Springer Nature remains neutral with regard to jurisdictional claims in published maps and institutional affiliations.

Ready to submit your research? Choose BMC and benefit from:

- fast, convenient online submission

- thorough peer review by experienced researchers in your field

- rapid publication on acceptance

- support for research data, including large and complex data types

- gold Open Access which fosters wider collaboration and increased citations

- maximum visibility for your research: over $100 \mathrm{M}$ website views per year

At BMC, research is always in progress.

Learn more biomedcentral.com/submissions 